\title{
The DynaGUI package
}

\section{DOI: $10.21105 /$ joss. 01942}

\section{Software}

- Review ¿

- Repository ¿

- Archive ${ }^{x}$

Editor: Mark A. Jensen ¿ Reviewers:

- @Chilipp

- @pythonpanda2

Submitted: 01 November 2019 Published: 28 December 2020

\section{License}

Authors of papers retain copyright and release the work under a Creative Commons Attribution 4.0 International License (CC BY 4.0).

\section{Benjamin Edward Bolling ${ }^{1,2}$}

1 European Spallation Source ERIC 2 MAX IV Laboratory

\section{Summary}

At large research facilities and industrial complexes, there is a need for control system user interfaces. However, modern facilities also require continuous upgrading, maintenance, and development, which means that also the control systems need to be upgraded. In order to simplify the construction of control systems and diagnostics, the DynaGUI (Dynamic Graphical User Interface) package was created. The main idea of this package is to get rid of the middlehand coding needed between hardware and the user by supplying the user with a simple GUI toolkit for generating diagnostics- and control-system GUls in accordance with any user's need. Initially developed at MAX IV Laboratory (Tavares et al., 2014), the initial and main users for this package are control rooms at large-scale research facilities, such as particle accelerators.

In order to further enhance the user-friendliness of this package, a simple system of configuration files has been developed, enabling users to configure the applications using any plain text-editor. The DynaGUI package consists of three applications:

- DynaGUI TF, a true/false (boolean) dynamic control system,

- DynaGUI Alarms, a dynamic diagnostics system for continuously monitoring of the values for a set of attributes, and

- DynaGUI NV, a system for observing attributes' numerical-, string-, vector- or waveform values.

Each DynaGUI application's layout is designed for simplicity. At the top of each application is a combobox with the list of attributes defined. Below the combobox is a button called 'Edit DynaGUI,' which opens a window for configuring the DynaGUI. Below the 'Edit DynaGUI' button is the dynamic field, in which a dynamic control panel is generated. Below the dynamic control panel field is the DynaGUI status bar, showing the last action carried out, error messages, or if an alarm is active (for DynaGUI Alarms). Below the status bar are the load and save buttons for loading or saving DynaGUI configuration files. These also have a tool-tip function showing the last loaded or saved file (in the current session). The simplest method to launch DynaGUI is via its launcher (Launcher.py), in which the user can select a control system and either directly define the path to the configuration file or browse to it. If the field is left blank, DynaGUI will load with a predefined setup.

DynaGUI TF (True/False) is dynamical in the sense that the user can insert the name of any device's servers and attributes that, in current state, are True or False. The GUI then builds itself up by creating buttons for each device server that will display the boolean of the device in the combobox selected attribute. First, the application will try to connect to the device's server as entered, and then read in the attribute selected in the combobox and paint the button's background colour: green meaning True, red meaning False, fuchsia meaning attribute not existing or not boolean, and maroon meaning that the device cannot be connected. 
The DynaGUI Alarms (Dynamic Alarms GUI) has been designed to monitor numerical values and notify user(s) if a condition is not fulfilled. To edit the alarms GUI, the user has to press Edit DynaGUI to open an edit-panel. In the left window of the edit-panel, the user has to define the list of devices' server domains and signals to monitor. In the right window of the edit-panel, the user has to define descriptions of the different alarm signals as they should appear in the DynaGUI Alarms control panel, as well as the sweep-time (how frequently the system should check the values). The DynaGUI Alarms' dynamic panel can be divided into three columns for each device and attribute it monitors. The first column contains the descriptions of the alarms, the second column contains the numerical values from the last sweep, the fourth column contains the alarm limit, and the third column contains a combobox with two gaps (larger than or smaller than) which points in the way it should between the read value and the alarm limit. For gap criteria that are fulfilled, the background colour of the alarm description is painted lime-green. If a gap criteria is not fulfilled, the computer's speakers will emit an alarm, and the display will show a message in the DynaGUI Alarms' status bar. The alarm description's background colour becomes red.

DynaGUI NV is the most advanced tool in the package. Each device has two columns. The first column is a button with the server address of the device, whilst the value of the selected attribute is shown in the second column, enabling users to inspect values in a simple and fast manner. DynaGUI NV also allows for launching a controller for the device (using AtkPanel for Tango Controls (Chaize et al., 1999)) or for initializing 1D plots or 2D colormaps of the selected attribute for all devices for which the attribute is valid. Each device's control panel button becomes painted in lime-green if the attribute is valid, in fuchsia if the attribute is not existing for the device and maroon if a connection to the device cannot be established. The plot initialization automatically launches for all devices for which the attribute is valid. The plotting tool has been elaborately described in the DynaGUI documentation. Features in the 1D graph tool include plotting read values in real time, and setting up and plotting equations (or functions) by using NumPy (van der Walt et al., 2011) of the read values as well as combining the read values with one another. Examples are shown in Figure 1. The 1D graph tool supports both scalar and vector (or waveform) plotting in real time, and plot data can be saved and loaded.

The package aims to enable users to construct dynamic GUIs for multiple purposes, and the author is open to implementing new functions and control systems on demand. For testing purposes, an artificial control system 'Randomizer' has been created displaying only random values. In this DynaGUI version, the DynaGUI panels are constructed using PyQt (Riverbank Computing Limited, 2016), with versions 4 and 5 supported. The author is working on fully implementing the PyEPICS (PyEpics, 2014) package as well as a new Finance package built on Pandas (McKinney, 2011) and Matplotlib (Hunter, 2007) in order to have more sources to monitor live-stream data from and hence to demonstrate the openness that serves as the core of the package. 


\section{Figures}

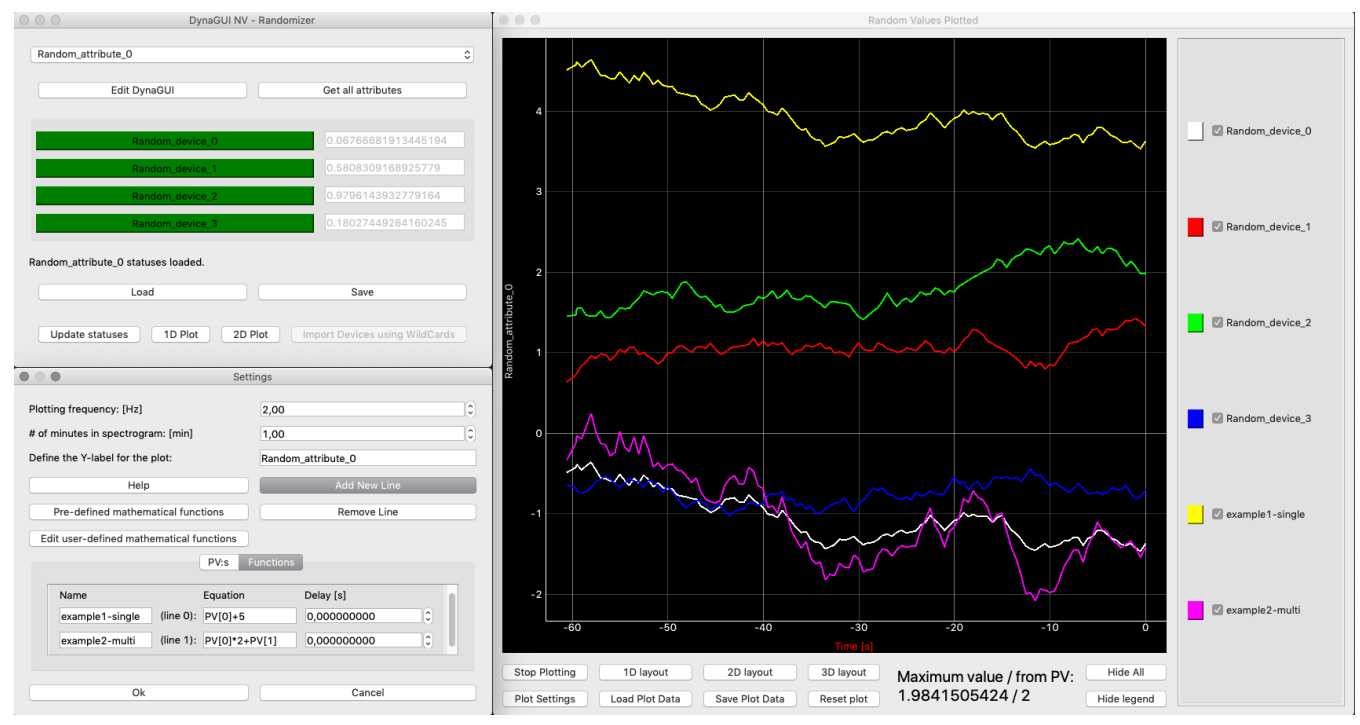

Figure 1: A dynamic control panel of DynaGUI NV has been configured (top-left), from which a 1D realtime plot has been launched for 4 artificial devices for a made-up attribute (right). Using this tool, two other lines have been set up as functions of two input data streams, with equations defined in the bottom-left figure and then plotted.

\section{Acknowledgements}

The author wants to thank Bernhard Meirose at the MAX IV Laboratory for the discussion that inspired and led me into developing this package. The author also recognises and wants to thank Jonas Petersson and Robin Svard at the MAX IV Laboratory for developing the original 2D Spectrogram Application (for monitoring transverse beam position via Beam Position Monitors at the MAX IV Laboratory storage rings). The author also wants to thank Bernhard Meirose at the MAX IV Laboratory for giving the inspiration and awakening the idea to create this package.

\section{References}

Chaize, J. M., Gotz, A., Klotz, W.-D., Meyer, J., Perez, M., \& Taurel, E. (1999). TANGO an object oriented control system based on CORBA. ICALEPCS'99.

Hunter, J. D. (2007). Matplotlib: A 2D graphics environment. Computing in Science \& Engineering, 9(3), 90-95. https://doi.org/10.1109/MCSE.2007.55

McKinney, W. (2011). Pandas: A foundational python library for data analysis and statistics. Python for High Performance and Scientific Computing, 14.

PyEpics: Interface for the Channel Access (CA) library of the EPICS Control System to the Python Programming language. (2014). https://cars9.uchicago.edu/software/python/ pyepics3/

Riverbank Computing Limited. (2016). PyQt5: Python bindings for the Qt cross platform UI and application toolkit. https://www.riverbankcomputing.com/software/pyqt/ 
JesS

Tavares, P. F., Leemann, S. C., Sjostrom, M., \& Andersson, A. (2014). The MAX IV storage ring project. Journal of Synchrotron Radiation, 21. https://doi.org/10.1107/ S1600577514011503

van der Walt, S., Colbert, S. C., \& Varoquaux, G. (2011). The NumPy array: A structure for efficient numerical computation. Computing in Science Engineering, 13(2), 22-30. https://doi.org/10.1109/MCSE.2011.37 